\title{
ON ITERATES OF CONTINUOUS FUNCTIONS ON A UNIT BALL
}

\section{HASKELL COHEN AND JACK HACHIGIAN}

Let $B^{n}$ be the unit ball in $R^{n}$, Euclidean $n$-space, i.e. $B^{n}$ $=\left\{x: x \in R^{n}, d(x, 0) \leqq 1\right\}$. If $f$ and $g$ are any two functions of $B^{n}$ to itself define, as usual, $\|f-g\|=\sup \left\{d(f(x), g(x)): x \in B^{n}\right\}$. J. Ax [1] has conjectured that if $f$ is a continuous function of $B^{n}$ on to itself such that $f$ restricted to the boundary is the identity, then $\left\|f^{(m+1)}-I\right\|$ $\geqq\left\|f^{(m)}-I\right\|$ for $m=1,2, \cdots$, where $I$ is the identity and $f^{(m)}$ is the $m$ th iterate of $f$, e.g. $f(f(x))=f^{(2)}(x)$.

Theorem 1 below shows the conjecture is true for $n=1$. Theorem 2 shows the conjecture is false for $n=2$. A concluding comment disposes of the cases $n>2$.

THEOREM 1. Let $f$ be a continuous function on $[-1,1]$ to itself such that $f(-1)=-1$ and $f(1)=1$. Then $\left\|f^{(m+1)}-I\right\| \geqq\left\|f^{(m)}-I\right\|$.

Proof. The theorem is trivially true when $f$ is the identity so we will only consider the case where $f \neq I$. The proof is by induction.

Let $G=\{x: f(x)=x\}$. The complement of $G$ is a collection of disjoint open subintervals $S_{\alpha}$ of $[-1,1]$. On each $S_{\alpha}$, either $f(x)>x$ or $f(x)<x$ for all $x \in S_{\alpha}$. If we let $f^{(0)}=I$, the first step of the induction is clear. (One could start out at $f^{(1)}=f$, but it is not necessary.) Assume then that for $k=1,2,3, \cdots, m$ we have $\left\|f^{(k)}-I\right\|>\left\|f^{(k-1)}-I\right\|$. Since $[-1,1]$ is compact, we know there exists $r \in[-1,1]$ such that $\left|f^{(m)}(r)-r\right|=\left\|f^{(m)}-I\right\|$. We assume $f^{(m)}(r)>r\left(f^{(m)}(r)<r\right.$ is argued analogously) and show $f(r)>r$. It is clear that $f(r) \neq r$. If $f(r)<r$ then for $s=f(r)$ we have

$$
\begin{aligned}
\left\|f^{(m-1)}-I\right\| & \geqq\left|f^{(m-1)}(s)-s\right| \\
& =\left|f^{(m)}(r)-s\right|=f^{(m)}(r)-s \\
& >f^{(m)}(r)-r=\left\|f^{(m)}-I\right\|,
\end{aligned}
$$

a contradiction. Hence $r \in S_{\alpha}$ on which $f(x)>x$ for all $x \in S_{\alpha}$. If $S_{\alpha}$ $=(a, b)$ then $f(a)=a<r<b=f(b)$, and the continuity of $f$ insures the existence of $q \in S_{\alpha}$ such that $f(q)=r$. Moreover, $q<r$ since $f(x)>x$ on $S_{\alpha}$. Now,

$$
\begin{aligned}
\left\|f^{(m+1)}-I\right\| & \geqq\left|f^{(m+1)}(q)-q\right| \\
& =f^{(m)}(r)-q>f^{(m)}(\boldsymbol{r})-r=\left\|f^{(m)}-I\right\|
\end{aligned}
$$

Received by the editors January 12, 1966. 
which completes the proof. We note that if $f$ is not the identity we have actually shown that $\left\|f^{(m)}-I\right\|>\left\|f^{(m-1)}-I\right\|$.

TheOREM II. Let $n \geqq 2$. Then there exist certain maps satisfying the conditions in the initial paragraph for which $\left\|f^{(k)}-I\right\|<\|f-I\|$.

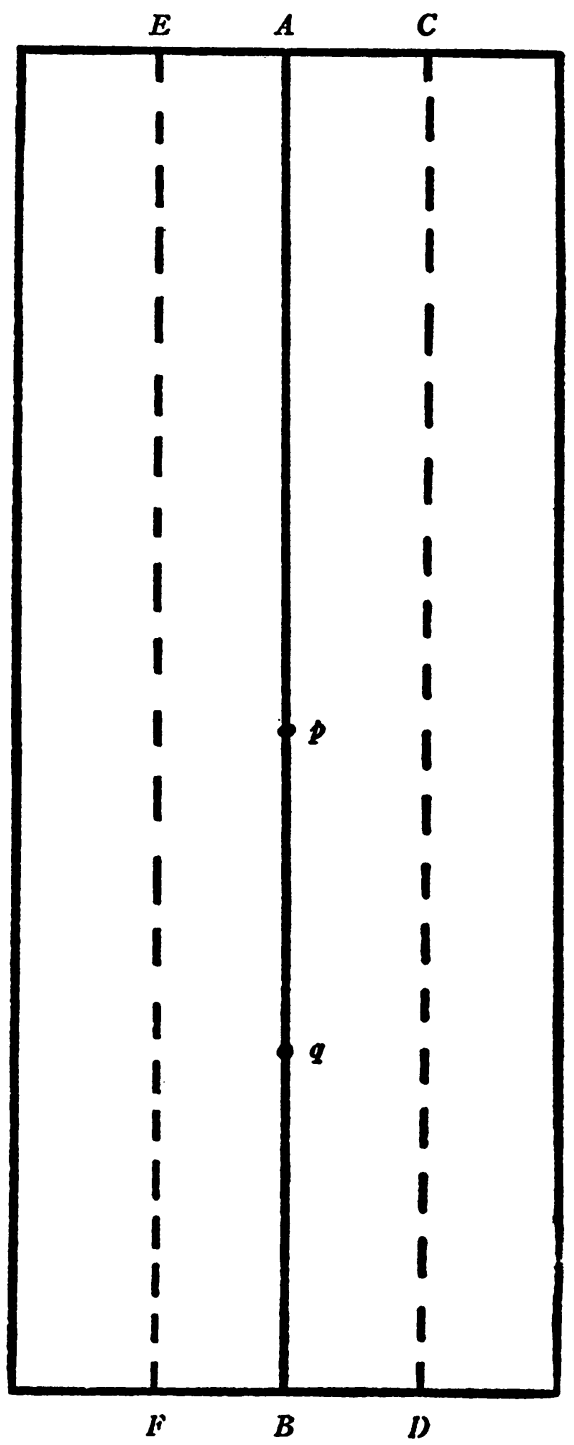

Figure 1 
Proof. Consider a rectangular strip $S=\{(x, y):-a \leqq x \leqq a, 0 \leqq y$ $\leqq 1\}$ (Figure I) where $a$ is some positive number. Let $p$ be the point in $S$ having co-ordinates $(0,1 / 2)$ and $q \in S$ having co-ordinates $(0,1 / 4)$. These choices are arbitrary but they help fix ideas. Let $A B$, $C D, E F$ be the vertical lines in $S$ such that $x=0, a / 2$, and $-a / 2$ respectively. Let $g: S \rightarrow S$ where $g(x, y)=\left(x, y^{2-|x| / a}\right)$. This defines a flow which is continuous (even $C^{\infty}$ ), which is the identity on the boundary, and where all interior points flow downward on a vertical line.

Now consider a plane set $Z$ illustrated in Figure II, and let the dis-

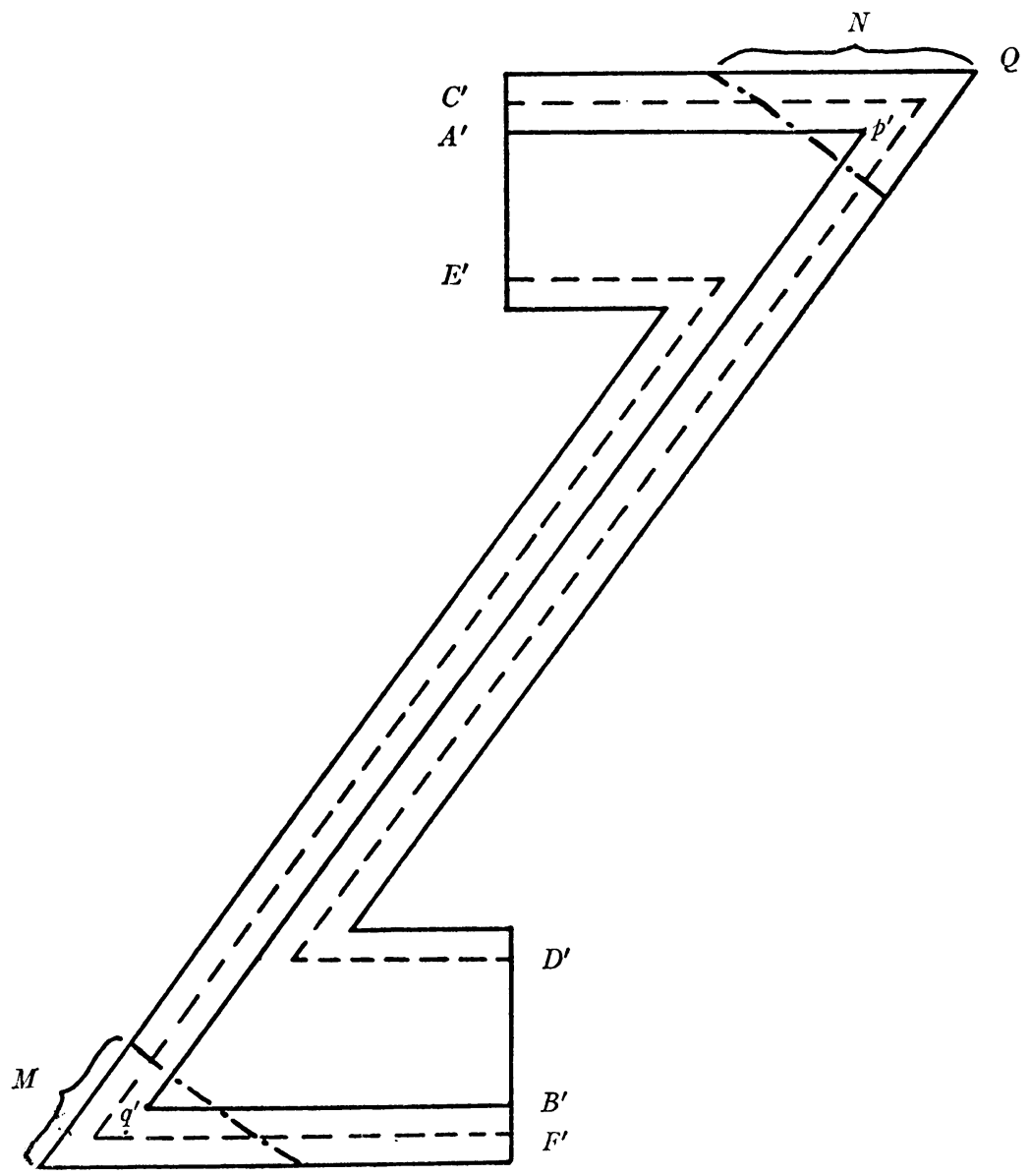

Figure II 
tance between the points labeled $Q$ and $R$ be $r$. Choose a conveniently small positive number $\epsilon$, and define $N$ to be the set of all points in $Z$ whose distancie from $R$ is greater than $r-\epsilon$, and define $M$ as the set of points in $Z$ having distance from $Q$ greater than $r-\epsilon$. Clearly if $x, y \in Z$ and $d(x, y)>r-\epsilon$ then $x \in N$ and $y \in M$, or conversely. Select $p^{\prime}$ interior to $N$ and $q^{\prime}$ interior to $M$ so that $d\left(p^{\prime}, q^{\prime}\right)>r-\epsilon$. Now, let $h$ be a homeomorphism of $S$ onto $Z$ taking $p \rightarrow p^{\prime}$ and $q \rightarrow q^{\prime}$ and the lines $A B, C D, E F$, respectively on to the broken lines $A^{\prime} B^{\prime}, C^{\prime} D^{\prime}$, and $E^{\prime} F^{\prime}$ as indicated in Figure II. Note, in particular, that the image of $C D$ is disjoint from $\bar{M}$ while the image of $E F$ misses $\bar{N}$, where the bar indicates the closure of the set.

Consider the set $Z$ imbedded in the interior of $B^{2}$ and define $f: B^{2} \rightarrow B^{2}$ by

$$
\begin{aligned}
& f(x)=x \quad \text { if } x \notin Z, \\
& f(x)=h g h^{-1}(x) \quad \text { if } x \in Z .
\end{aligned}
$$

Since $g$ restricted to the boundary of $S$ is the identity, $f$ restricted to the boundary of $Z$ is the identity, and hence $f$ is continuous-in fact, is a homeomorphism. Moreover, $\|f-I\| \geqq d\left(f\left(p^{\prime}\right), p^{\prime}\right)=d\left(q^{\prime}, p^{\prime}\right)>r-\epsilon$. Now, notice that the image of $C D$ cuts $N$ into two components, say $U$ and $V$, where $U$ is the component containing $p^{\prime}$ and $V$ the other. For $x \in U$, the sequence $\left\{f^{(i)}(x)\right\}$ converges to some point on the line segment $D^{\prime} F^{\prime}$; hence there is an integer $k$ such that if $i \geqq k, f^{(i)}(x) \notin M$ for all $x \in U$. Now, since for $x \notin N, d\left(f^{(i)}(x), x\right) \leqq r-\epsilon$, and for $x \in V$, $d\left(f^{(i)}(x), x\right)<r-\epsilon$, and for $x \in U, d\left(f^{(k)}(x), x\right) \leqq r-\epsilon$, we have

$$
\left\|f^{(k)}-I\right\| \leqq r-\epsilon<\|f-I\| .
$$

Therefore, the sequence $\left\{\left\|f^{(m)}-I\right\|\right\}$ cannot be monotone increasing.

This proof can, of course, be extended to dimensions greater than 2 by applying $f$ on the first two co-ordinates of a point and leaving the other co-ordinates fixed.

The reader should note that in the cases $n \geqq 2$ a 1 -dimensional construction has been used. In fact every subset of $B^{n}$ is homeomorphic to $B^{1}$ by a map which preserves order relations between distances if $n=1$. This is not true for $B^{n}, n \geqq 2$, as the $Z$-shaped figure shows.

\section{REFERENCE}

1. J. Ax, Oral communication.

Louisiana State University and

State University of New York at Stony Brook 